\title{
SOX10 as an immunohistochemical marker in cancer diagnostics
}

\author{
Beata Pytlak1,2, Monika Prochorec-Sobieszek 1,2, Anna Szumera-Ciećkiewicz ${ }^{1,2}$ \\ 'Institute of Hematology and Transfusion Medicine, Diagnostic Hematology Department, Warsaw, Poland \\ ${ }^{2}$ Maria Skłodowska-Curie Institute - Oncology Center, Department of Pathology and Laboratory Diagnostics, Warsaw, Poland
}

SOX10 is a transcription factor that plays an important role in the formation, specification and differentiation of neural crest cells. Recent studies indicate that this protein is expressed in tumors of the nervous system, salivary glands, breast, soft tissues and melanoma cells. Due to its higher sensitivity and specificity in comparison to other available markers, SOX10 protein is mainly used in the diagnosis of malignant peripheral nerve sheet tumor and primary and metastatic melanoma including desmoplastic type. SOX10 has a more readable immunohistochemical staining, which also facilitates histopathological interpretation. Several monoclonal and polyclonal antibodies for diagnostics are currently available. Unification of anti-SOX10 antibodies to increase specificity, eliminate variability and strength of immunohistochemical reactions will support its diagnostic usefulness.

NOWOTWORY J Oncol 2019; 69, 2: 58-64

Key words: SOX10 protein, immunohistochemistry, melanocytic marker, malignant peripheral nerve sheath tumor, anti-SOX10 antibodies

\section{Proteins from the SOX family}

The sex-determining region $Y$ (SRY) gene responsible for the male sex that was found on the $Y$ chromosome gave rise to the class of genes called SOX. The name of SOX [SRY-related high-mobility group ( $\mathrm{HMG}$ ) box] indicates that the class of genes has a common construction of the high mobility group (HMG) domain. The HMG domains in all SOX proteins have a similar structure and all are thought to be capable of binding to the same DNA sequence - AACAA (A / T) G. The family of SOX genes is indispensable in many processes including: the development of the nervous and immune systems, the formation of pigment cells, sex determination, skeletal genesis and in the development of the eyes [1]. SOX proteins, due to their ability to bind DNA through the HMG domain, are included in DNA-binding proteins. In mammals, the family of SOX proteins consists of about 20 transcription factors. Nine groups of SOX proteins have been isolated according to transcription factors content: A, B1, B2, C, D, E, F, G and H. It has been proven that each group includes proteins showing $>80 \%$ of similarity in the HMG domain construction. SOX10 together with SOX9 and SOX8 form a group of SOXE transcription factors [1, 2].

\section{SOX10 structure and functions}

SOX10 is a transcription factor encoded by the SOX10 gene located on the long arm of chromosome 22 at position 22q13.1 and encodes 446 amino acids. SOX10 plays a key role in the development of testes, oligodendrocytes, the central nervous system and chondrocytes [3]. The regulation of SOX10 functions consists in binding this protein to promoters or enhancers of transcription in target genes, alone or in combination with other transcription factors $[4,5]$. SOX10 is responsible for a number of important functions in the body. Its crucial role is participation in maintaining the pluripotency of progenitor cells via the specification and differentiation of cell lines and the formation of neural crest cells. The products of these cells include: neural and glial cells in the peripheral nervous system, 
skin melanocytes, thyroid gland, adrenal medulla and visceral sacroiliac cartilage [6-8]. Additionally, SOX10 is essential in the process of central and peripheral nervous system myelination. In Schwann cells, SOX10 regulates the transcription of myelin proteins, such as zero proteins, peripheral myelin 22 and connexin $32[9,10]$. Another function of SOX10 is the regulation of lipophilin and basic myelin protein transcription in oligodendrocytes. According to recent studies, SOX10 is a regulator of c-RET - a protein tyrosine kinase receptor that plays a central role in the development of the intestinal nervous system. SOX10 is also indispensable in the process of regulating the transcription of genes necessary for the synthesis of melanin, such as: DOPA chromium tautomerases, tyrosinase and tyrosinase-related protein [1, 11-15]. Figure 1 presents the location and major functions of the SOX10 gene.

The germline SOX10 mutations were identified in the Waardenburg-Shah syndrome type II and type IV. These mutations include mainly reading frame shift, nonsense type and one splicing site $[6,16]$. The Waardenburg-Shah syndrome is characterized by the presence of bilateral deep hearing loss and pigment abnormalities. The irregularities associated with the depigmentation include: white hair, blue iris with gray spots and skin discoloration $[6,16,17]$. Type IV ofWaardenburg-Shah syndrome is the rarest of four main types. It occurs with a frequency < 1 per 1000000 and so far only about 100 cases were reported [18]. SOX10 mutations were also detected in a more severe syndrome characterized by the association of the features ofWaardenburg-Shah syndrome with peripheral demyelinating neuropathy-central dysmyelinating leukodystrophy and Hirschsprung disease $(\mathrm{PCWH})$, which is a congenital functional disorder of the gastrointestinal motility due to the lack of intestinal nerve ganglia $[19,20]$. In the autosomal dominant form of Kalmann's syndrome, the SOX10 loss of function mutations were found in about 38\% cases. The disease is characterized by congenital hypogonadotropic hypogonadism and anosmia or hyposmia [6]. The Yemenite deaf-blind hypopigmentation syndrome characterized by hypopigmentation, small cornea, coloboma and the occurrence of severe hearing loss is also caused by mutations in the SOX10 gene [21].

It is important that SOX 10 together with the NOTCH1 protein influence proliferation and resistance to radiation in many cancer stem cells i.e. prostate cancer [22]. Selective expression of SOX10 in normal tissues, i.e. peripheral nerves, glandular cells in salivary or sweat glands in comparison with highly specific immunohistochemical staining in melanoma, schwannoma, malignant peripheral nerve sheath tumor, myoepithelial, and some salivary gland tumors make this protein useful in the routine histopathological diagnostics [23, 24]. Currently, a large selection of anti-SOX10 antibodies is available, including poly - and monoclonal clones. The authors present the importance of SOX10 in the histopathological diagnostics and the review of antibodies used in the immunohistochemical assessment.

\section{SOX10 as a melanocytic marker}

Melanoma is a deadly skin disease known for its aggressive course and high risk of metastasis. This disease may show a wide range of morphological features, which gives rise to difficulties in interpretation. Diagnosis of melanoma still remains complicated, because ambiguous melanocytic changes may show features that overlap with melanoma and benign changes. Therefore, accurate clinical diagnosis in patients is highly relevant to further clinical management [25].

SOX10 is one of the recently known immunohistochemical markers useful in the diagnosis of tumors arising from melanocytes. It directly affects the microphthalmia-associated transcription factor (MITF), which regulates the transcription transctiption responsible for melanin synthesis and survival of postnatal melanocytes. SOX10 also influences the expression of other genes that mediate melanin synthesis [26-28]. In several publications SOX10 was shown as a highly sensitive
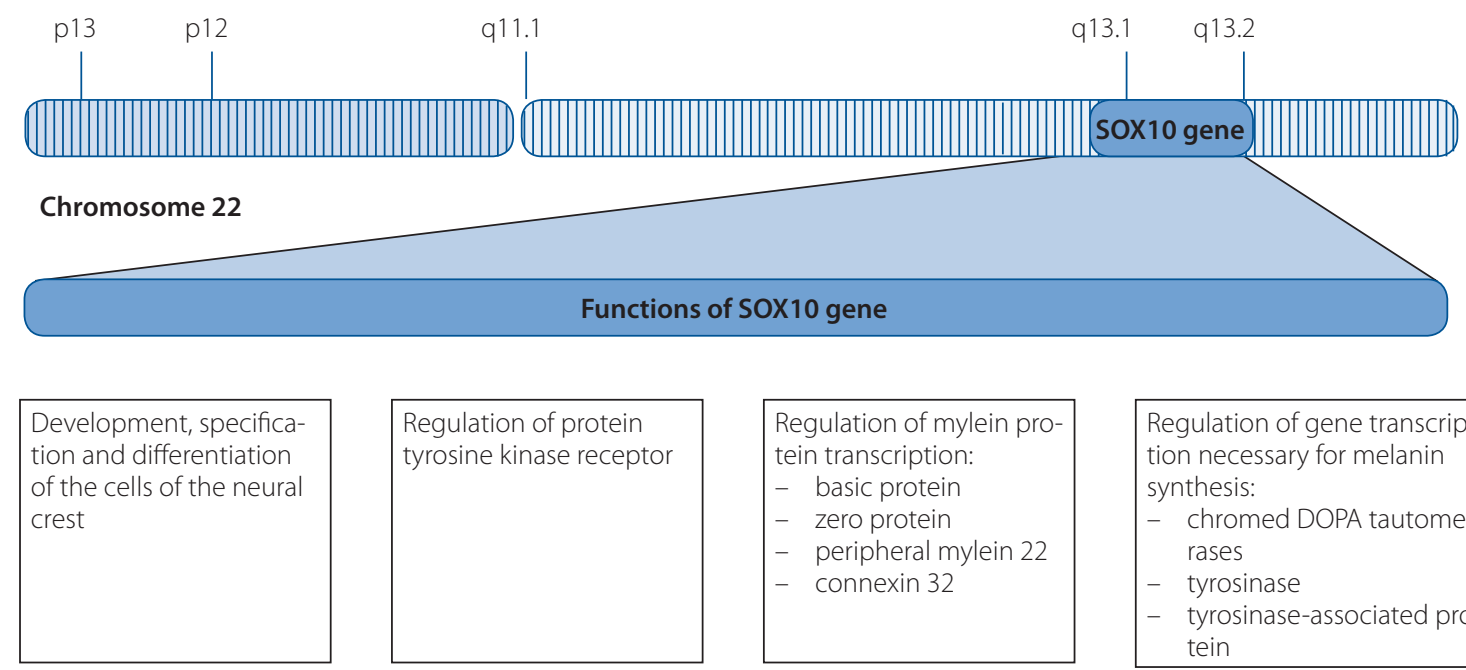

Regulation of mylein pro-

tein transcription:

- basic protein

zero protein

- peripheral mylein 22

- connexin 32
Regulation of gene transcription necessary for melanin synthesis:

- chromed DOPA tautomerases

- tyrosinase

- tyrosinase-associated protein

Figure 1. Chromosome location and major functions of the SOX10 gene 
and specific melanocytic marker, which can be used in the diagnosis of melanoma $[24,29]$. The first study, in which the expression of SOX10 in immunohistochemistry in melanomas was studied, was the research work of Gershon et al. The presence of positive immunohistochemical reactions was noted in all analyzed melanoma cells [30].

In 2014, Mohamed et al. also confirmed 100\% expression of SOX10 in melanoma stem cell. High SOX10 expression excluded the possibility of using this protein as a prognostic marker, but its diagnostic utility was evaluated [31]. SOX10 protein showed $100 \%$ expression in primary cutaneous and capsular lymph node nevi, 95-100\% in primary and 97-100\% in metastatic melanoma lesions [24,32,33]. Moreover, the presence of high expression of SOX10 in desmoplastic melanoma cells has also been demonstrated [23, 24, 34]. In addition, it was proven that the expression of SOX10 was stronger and more homogenic than the $\mathrm{S} 100$ staining. Karamchandani and et al. found positive expression of SOX10 and S100 in 78\% and $57 \%$ in desmoplastic melanoma respectively [23]. Ramos-Herberth et al. showed a significant difference of the staining intensity between desmoplastic melanoma cells - high and strong expression, and fibrocytes/spindle histiocytes - weak or no expression of SOX10 protein. The results indicated that immunohistochemical evaluation of SOX10 can be used to differentiate desmoplastic melanomas from post-traumatic scars as well [34].

A large number of reports regarding very high sensitivity of the SOX10 protein in melanoma may indicate that it is a good marker in the diagnosis of this neoplasm. In contrast to the S100 marker, SOX10 is not expressed in dendritic cells. Limited positive background effect indicates that SOX10 might be even a better diagnostic marker [29]. The expression of SOX10 protein can also be very helpful in the diagnosis of melanoma metastases to sentinel lymph nodes due to its high sensitivity and lack of expression in dendritic cells [35]. SOX10 may be used in differential diagnosis of melanoma in situ, as well as solar keratosis with melanocytic hyperplasia [36].

Reviewed studies on SOX10 expression were based on heterogeneous groups of tissues, sometimes without statistical significance. In addition, the comparative analysis to other well-known melanoma markers, including S100, HMB45 or Melan A was limited. The lack of a large scale comparative research still does not allow for defining recommendation of SOX10 as a first-line marker or at least an equivalent to S100 in the diagnosis of melanoma.

\section{SOX10 in tumors of the nervous system}

Central nervous system (CNS) tumors are a heterogeneous group of neoplasms constituting about 3\% of all cancers, and they are responsible for $7 \%$ of all cancer deaths. In the nervous system, positive expression of SOX10 is evident in oligodendrocytes, sensory and sympathetic ganglia, Schwann cells and intestinal cells. The key function of SOX10 in the nervous system is its involvement in the development of Schwann cells and the effect on the differentiation of oligodendrocytes in the brain and spinal cord. Different patterns of SOX10 protein expression in CNS tumors are reported. The results showed $100 \%$ expression of SOX 10 in patients with diagnosed Schwannoma tumors, 98-100\% in neurofibromas, and much lower, 50-55 \% in malignant peripheral nerve sheath tumor (MPNST) $[24,30,37]$

MPNST is a rare malignant tumor with an aggressive course constituting 2\% sarcomas derived from soft tissues. This malignancy may occur in type I neurofibromatosis after radiotherapy or sporadically [38]. In the diagnosis of MPNST, the $\mathbf{S 1 0 0}$ protein is routinely used as a stable and specific marker. Current studies showed that the expression of the SOX10 protein is comparable to the $\mathbf{S 1 0 0}$ protein in patients suffering from neurofibroma and schwannoma. Nonaka and et al. presented that SOX10 may be a more sensitive and specific marker of MPNST; 49\% vs. 30\% was positive to SOX 10 and S100 respectively. Moreover, it was also confirmed that granular cell tumors are associated with neoplastic nerve tumors that come together with the neural crest [24]. In addition, it was noted that the expression of SOX10 protein is almost always absent in tumors with which MPNST can be misdiagnosed [23]. It was confirmed that the evaluation of SOX10 strongly supports the differential diagnostics of MPNST from synovial sarcoma $[24,37]$.

\section{SOX10 in other malignancies}

The presence of SOX 10 was also demonstrated in salivary gland, breast and soft tissue tumors. Due to limited number of studies, the expression of SOX10 in epithelial tumors has still not been well established. SOX10 is rarely expressed in epithelial tumors with the exception of the salivary glands tumors, in which the presence of SOX10 has been confirmed by numerous studies $[23,24,39,40]$. Nonaka et al. confirmed that SOX10 protein is expressed in myoepithelial cells [24]. The normal acinar and myoepithelial cells and tumors originating from those cells are SOX10 positive $[23,40]$. Recently, a large group of patients with different salivary gland tumors was evaluated; the SOX10 expression depended on the histopathological type and presented as below: multiform adenoma - 79\%, monomorphic adenomas - 100\%, polymorphic adenocarcinoma of low malignancy $88 \%$, adenoid cystic adenocarcinoma - 96\% and adenocarcinomas - 96\%. Moreover, there was no expression of the SOX10 protein in the other subtypes of salivary gland tumors; which means that the determination of the SOX10 protein expression may be a good marker supporting the differential diagnosis of some tumors subtypes $[40,41]$. A few studies reported positive SOX10 expression in pheochromocytomas, PEComas and carcinoid carcinomas [42]. In breast cancer there was also a positive expression of SOX10 [43]. Cimino-Mathews et al. confirmed its occurrence in patients with triple-negative or metaplastic breast cancer [39]. Malignant gastrointestinal neuroectodermal tumor, 
which was first described as an 'osteoclast-rich tumor of the gastrointestinal tract with features resembling clear cell sarcoma of soft parts' in 2003, is also SOX10 positive even though it lacks melanocytic differentiation [44]. Moreover, SOX genes probably play important roles in sarcoma cell pathogenesis, growth, and proliferation i.e.: SOX2 and SOX9 genes in osteosarcoma, chondrosarcoma and chordoma, SOX2, SOX6, and SOX17 genes in Ewing's sarcoma, SOX2, SOX9, and SOX10 genes in synovial sarcoma, SOX2 gene in fibrosarcoma, and SOX21 gene in liposarcoma [45]. In Figure 2, SOX10 expression in different tissues and tumors is presented. The frequency of positive SOX10 staining in soft tissue tumors is summarized in Table I.

\section{Anti-SOX10 antibodies in diagnostics}

Immunohistochemical staining of SOX10 is possible due to the high availability of anti-SOX10 antibodies offered by the diagnostics market. Currently several monoclonal and polyclonal antibodies are available, which are used for staining fixed and paraffin-embedded tissue material. The antibodies approved for routine diagnostics are produced by Cell Marque, Santa Cruz Biotechnology and Sigma Aldrich. Clones of monoclonal antibodies used in the diagnosis of SOX10 protein are mainly A2, 1E6, EP268 and SP267. They are rabbit and mouse antibodies. At present, the polyclonal anti-SOX10 antibody employed in routine diagnostics is the anti-SOX10 polyclonal rabbit antibody offered by Cell Marque. In medical databases, different polyclonal antibodies, which have been used, might be found $[46,47]$. The majority of published studies in immunohistochemistry staining utilized the Santa Cruz N-20 goat polyclonal antibody [23, 24]. Nevertheless, this antibody has been discontinued, and the new replacement is lgG1 monoclonal immunoglobulin with the A-2 clone. The manufacturer ensures that the new antibody is more specific, shows a stronger intensity and eliminates the variation in staining characteristic of each series of polyclonal antibodies. Recent literature has already revealed researches using the above-mentioned mouse monoclonal antibody $\lg \mathrm{G} 1[48,49]$.

Nevertheless, there are some controversies regarding the localization of SOX10 protein detection. Despite the fact that in most studies SOX10 staining shows a nuclear or nuclear reaction and weakly cytoplasmic staining, some reports show the perinuclear and cytoplasmic reactions $[23,24,46]$. The reason for these differences is the type or clone of antibody which is used. In studies where SOX10 was nuclear or nuclear and weakly cytoplasmic, a goat N-20 polyclonal antibody from Santa Cruz Biotechnology and a polyclonal rabbit one from Cell Marque were used $[24,47]$. In contrast, in the study, where the peri-nuclear and cytoplasmic reaction was shown, the Abcam anti-polyclonal rabbit antibody was applied. The authors interpreted the reaction as positive, which meant the presence of the SOX10 protein around nucleus and in cytoplasm. This protein probably appeared in these areas in the deactivated state [46].

\section{Summary}

1. Selective and specific expression of SOX10 in the melanocytic lesions including melanoma, nervous and salivary glands tumors is reported.

2. The immunohistochemical staining of SOX10 may be helpful in the routine histopathological diagnostics of the above malignancies.

3. SOX10 may be the major or alternative marker for differential diagnosis of melanoma from other lesions. The occurrence of a clear and extensive nuclear reaction facilitates the interpretation of the staining result.

4. SOX10 staining improves the diagnosis of desmoplastic melanoma and MPNST due to its higher sensitivity and specificity in comparison with other available markers.

5. In contrast to the $\mathbf{S 1 0 0}$ protein, the anti-SOX10 antibody does not demonstrate a positive staining result in dendritic cells, which facilitates its interpretation in lymph node evaluation.

6. A large scale research on the SOX10 protein expression in comparison to other melanocytic markers in sentinel lymph nodes is still needed, since there are no direct recommendations.

\section{Conflicts of interests: none declared}

This work has been implemented using the Project infrastructure POIG.02.03.00-14-111/13 funded by Operational Programme Innovative Economy 2007-2013, Priority II. R\&D Infrastructure, Measure 2.3. Investments connected with development of IT infrastructure of Science.

Table I. The frequency of positive SOX10 staining in soft tissue tumors; the summary has been prepared on the basis of the following publications: 23, 24, $30,47,50-52$

\begin{tabular}{lcc|}
\hline Histopathological type & Total number SOX10(+) cases & $\%$ SOX10(+) cases \\
\hline Schwannoma & $190 / 197$ & $97 \%$ \\
\hline Neurofibroma & $134 / 137$ & $98 \%$ \\
\hline Granular cell tumor & $143 / 153$ & $93 \%$ \\
\hline Malignant peripheral nerve sheath tumor & $245 / 346$ & $71 \%$
\end{tabular}





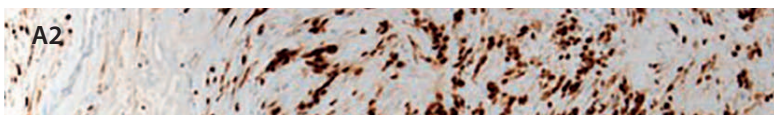



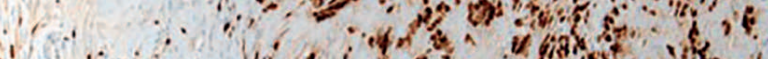

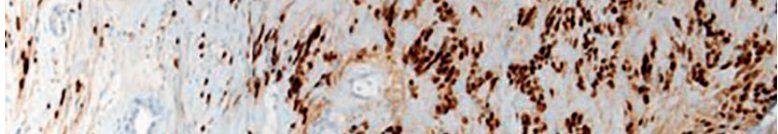

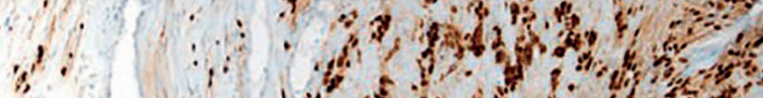





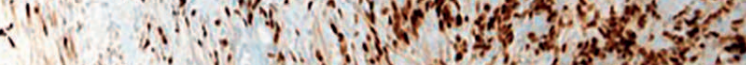

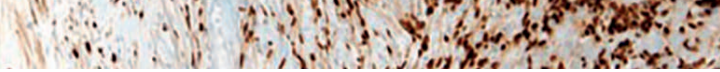
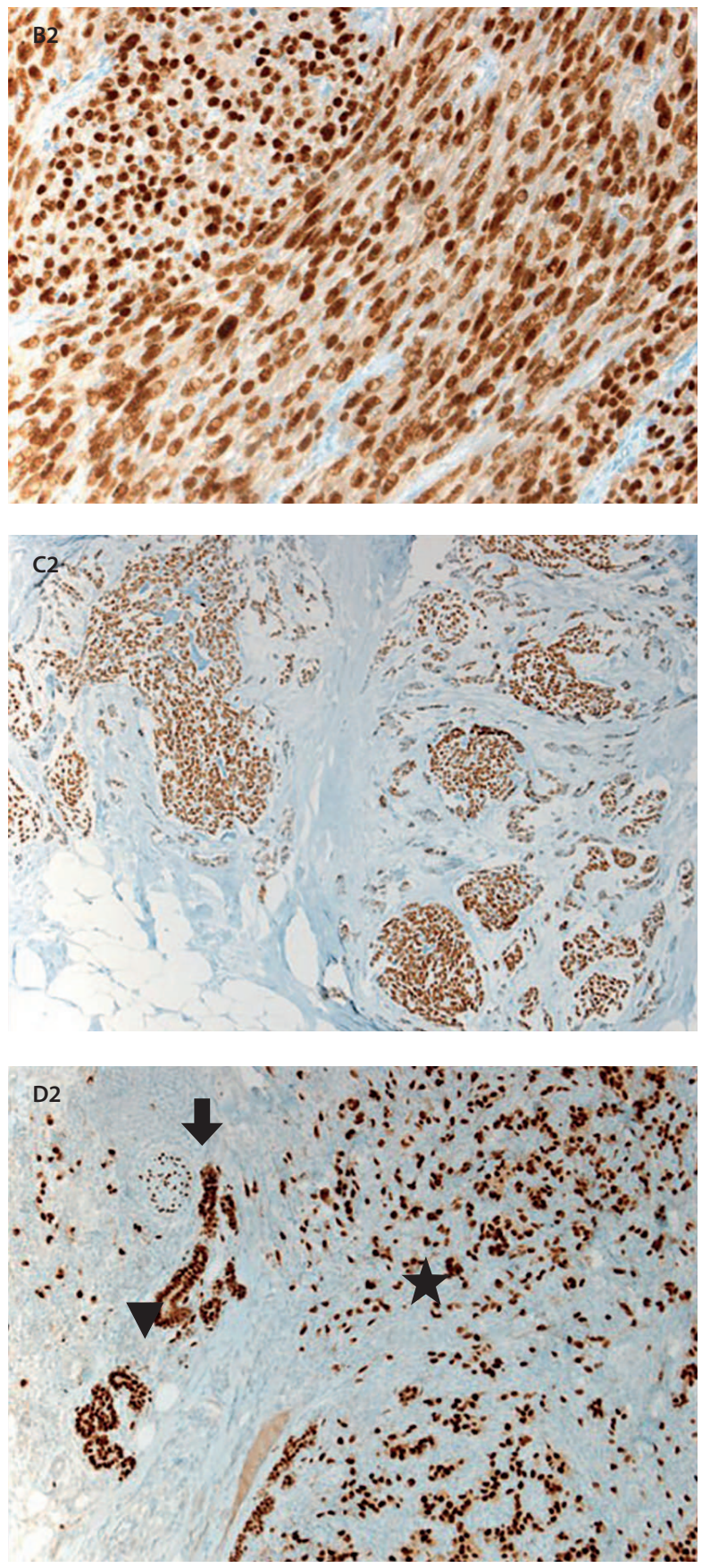

Figure 2. SOX10 immunostaining in different tumors. Schwannoma (100 x): A1 - HE, A2 - SOX10; MPNST (200 x): B1 - HE, B2 - SOX10. Myoepithelial carcinoma of breast, epithelioid neoplastic cells arranged in nests and trabelular pattern with abundant myxoid matrix: (40 x): C1 - PAS + Alcjan, C2 SOX10. Invasive melanoma of skin (100 x), arrow: nerve, arrowhead: sweat ducts, star: melanoma: D1 - HE, D2 - SOX10 

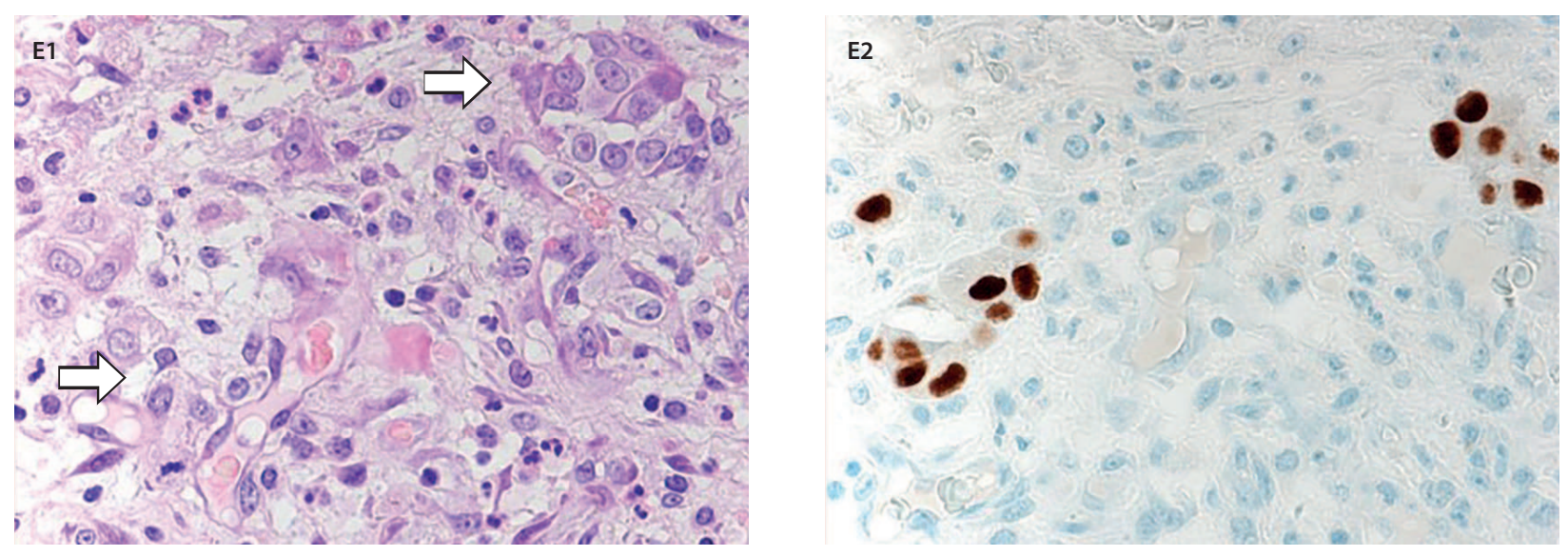

Figure 2 cont. Metastatic melanoma to lymph node, single neoplastic cells (600 x): E1 - HE, E2 - SOX10

\section{Anna Szumera-Ciećkiewicz}

Institute of Hematology and Transfusion Medicine

Diagnostic Hematology Department

ul. Indiry Gandhi 14

02-776 Warszawa, Poland

e-mail:szumann@gmail.com

\section{Received: 25 May 2019}

Accepted: 7 Jul 2019

\section{References}

1. Ordonez NG. Value of SOX10 immunostaining in tumor diagnosis. Advances in Anatomic Pathology 2013; 20: 275-283.

2. Sarkar A, Hochedlinger K. The sox family of transcription factors: versatile regulators of stem and progenitor cell fate. Cell Stem Cell 2013; 12: 15-30.

3. Falah N, Posey JE, Thorson W et al. 22q11.2q13 duplication including SOX 10 causes sex-reversal and peripheral demyelinating neuropathy, central dysmyelinating leukodystrophy, Waardenburg syndrome, and Hirschsprung disease. American Journal of Medical Genetics Part A 2017; 173: 1066-1070.

4. Kuhlbrodt K, Herbarth B, Sock E et al. Sox10, a novel transcriptional modulator in glial cells. The Journal of Neuroscience 1998; 18: 237-250.

5. Kamachi Y, Cheah KS, Kondoh H. Mechanism of regulatory target selection by the SOX high-mobility-group domain proteins as revealed by comparison of SOX1/2/3 and SOX9. Molecular and Cellular Biology 1999; 19: 107-120.

6. Pingault $\mathrm{V}$, Bodereau V, Baral $\mathrm{V}$ et al. Loss-of-function mutations in SOX10 cause Kallmann syndrome with deafness. American Journal of Human Genetics 2013; 92: 707-724.

7. Dupin E, Calloni G, Real C et al. Neural crest progenitors and stem cells. Comptes Rendus Biologies 2007; 330: 521-529.

8. Huang $X$, Saint-Jeannet JP. Induction of the neural crest and the opportunities of life on the edge. Developmental Biology 2004; 275: 1-11.

9. Bondurand N, Girard M, Pingault $V$ et al. Human Connexin 32, a gap junction protein altered in the X-linked form of Charcot-Marie-Tooth disease, is directly regulated by the transcription factor SOX10. Human Molecular Genetics 2001; 10: 2783-2795.

10. Peirano RI, Goerich DE, Riethmacher D et al. Protein zero gene expression is regulated by the glial transcription factor Sox 10 . Molecular and Cellular Biology 2000; 20: 3198-3209.

11. Lang $D$, Chen F, Milewski $R$ et al. Pax3 is required for enteric ganglia formation and functions with Sox 10 to modulate expression of c-ret. The Journal of Clinical Investigation 2000; 106: 963-971.

12. Potterf SB, Mollaaghababa R, Hou L et al. Analysis of SOX10 function in neural crest-derived melanocyte development: SOX10-dependent transcriptional control of dopachrome tautomerase. Developmental Biology 2001; 237: 245-257.

13. Stolt CC, Rehberg $\mathrm{S}$, Ader M et al. Terminal differentiation of myelin-forming oligodendrocytes depends on the transcription factor Sox 10. Genes \& Development 2002; 16: 165-170.
14. Hou L, Arnheiter H, Pavan WJ. Interspecies difference in the regulation of melanocyte development by SOX10 and MITF. Proceedings of The National Academy of Sciences of the United States of America 2006; 103: 9081-9085.

15. Murisier F, Guichard S, Beermann F. A conserved transcriptional enhancer that specifies Tyrp1 expression to melanocytes. Developmental Biology 2006; 298: 644-655.

16. Bondurand N, Dastot-Le Moal F, Stanchina Let al. Deletions at the SOX10 gene locus cause Waardenburg syndrome types 2 and 4. American Journal of Human Genetics 2007; 81: 1169-1185.

17. Inoue K, Khajavi M, Ohyama T et al. Molecular mechanism for distinct neurological phenotypes conveyed by allelic truncating mutations. Nature Genetics 2004; 36: 361-369.

18. Chandra Mohan SLN. Case of Waardenburg Shah syndrome in a family with review of literature. Journal of Otology 2018; 13: 105-110.

19. Verheij JB, Sival DA, van der Hoeven JH et al. Shah-Waardenburg syndrome and PCWH associated with SOX10 mutations: a case report and review of the literature. EJPN 2006; 10: 11-17.

20. Sergi C. Hirschsprung's disease: Historical notes and pathological diagnosis on the occasion of the 100 (th) anniversary of Dr. Harald Hirschsprung's death. World Journal of Clinical Pediatrics 2015;4: 120-125.

21. Bondurand N, Kuhlbrodt K, Pingault V et al. A molecular analysis of the yemenite deaf-blind hypopigmentation syndrome: SOX10 dysfunction causes different neurocristopathies. Human Molecular Genetics 1999; 8: 1785-1789.

22. Panaccione A, Chang MT, Carbone BE et al. NOTCH1 and SOX10 are Essential for Proliferation and Radiation Resistance of Cancer Stem-Like Cells in Adenoid Cystic Carcinoma. Clinical Cancer Research 2016; 22: 2083-2095.

23. Karamchandani JR, Nielsen TO, van de Rijn M et al. Sox 10 and S100 in the diagnosis of soft-tissue neoplasms. AIMM 2012; 20: 445-450.

24. Nonaka D, Chiriboga L, Rubin BP. Sox10: a pan-schwannian and melanocytic marker. The American Journal of Surgical Pathology 2008; 32: 1291-1298.

25. Tetzlaff MT, Torres-Cabala CA, Pattanaprichakul P et al. Emerging clinical applications of selected biomarkers in melanoma. Clinical, Cosmetic and Investigational Dermatology 2015; 8: 35-46.

26. Hemesath TJ, Steingrimsson E, McGill G et al. microphthalmia, a critical factor in melanocyte development, defines a discrete transcription factor family. Genes \& Development 1994; 8: 2770-2780.

27. Yasumoto K, Yokoyama K, Shibata K et al. Microphthalmia-associated transcription factor as a regulator for melanocyte-specific transcription of the human tyrosinase gene. Molecular and Cellular Biology 1994; 14: 8058-8070.

28. Lee $M$, Goodall J, Verastegui $C$ et al. Direct regulation of the Microphthalmia promoter by Sox 10 links Waardenburg-Shah syndrome (WS4)-associated hypopigmentation and deafness to WS2. The Journal of Biological Chemistry 2000; 275: 37978-37983.

29. Blochin E, Nonaka D. Diagnostic value of Sox10 immunohistochemical staining for the detection of metastatic melanoma in sentinel lymph nodes. Histopathology 2009; 55: 626-628.

30. Gershon TR, Oppenheimer O, Chin SS et al. Temporally regulated neural crest transcription factors distinguish neuroectodermal tumors of varying malignancy and differentiation. Neoplasia 2005; 7: 575-584. 
31. Mohamed A, Gonzalez RS, Lawson D et al. Tumor stem cells (CD271, c-kit, SOX10) in Melanomas: prognostic and outcome implications. AIMM 2014; 22: 142-145.

32. Agnarsdottir M, Sooman L, Bolander A et al. SOX10 expression in superficial spreading and nodular malignant melanomas. Melanoma Research 2010; 20: 468-478.

33. Kandukuri SR, Lin F, Gui L et al. Application of Immunohistochemistry in undifferentiated neoplasms: a practical approach. Archives of Pathology \& Laboratory Medicine 2017; 141: 1014-1032.

34. Ramos-Herberth FI, Karamchandani J, Kim J et al. SOX10 immunostaining distinguishes desmoplastic melanoma from excision scar. Journal of Cutaneous Pathology 2010; 37: 944-952.

35. Jennings $\mathrm{C}$, Kim J. Identification of nodal metastases in melanoma using sox-10. The American Journal of Dermatopathology 2011; 33: 474-482.

36. Buonaccorsi JN, Prieto VG, Torres-Cabala C et al. Diagnostic utility and comparative immunohistochemical analysis of MITF-1 and SOX10 to distinguish melanoma in situ and actinic keratosis: a clinicopathological and immunohistochemical study of 70 cases. The American Journal of Dermatopathology 2014; 36: 124-130.

37. Kang Y, Pekmezci M, Folpe AL et al. Diagnostic utility of SOX10 to distinguish malignant peripheral nerve sheath tumor from synovial sarcoma, including intraneural synovial sarcoma. Modern Pathology 2014; 27: 55-61.

38. Ng VY, Scharschmidt TJ, Mayerson JL et al. Incidence and survival in sarcoma in the United States: a focus on musculoskeletal lesions. Anticancer Research 2013; 33: 2597-2604.

39. Cimino-Mathews A, Subhawong AP, Elwood H et al. Neural crest transcription factor Sox 10 is preferentially expressed in triple-negative and metaplastic breast carcinomas. Human Pathology 2013; 44: 959-965.

40. Hsieh M-S, Lee Y-H, Chang Y-L. SOX10-positive salivary gland tumors: a growing List, including mammary analogue secretory carcinoma of the salivary gland, sialoblastoma, Low-grade salivary duct carcinoma, basal cell adenoma/adenocarcinoma, and a subgroup of mucoepidermoid carcinoma 2016.

41. Lee $\mathrm{JH}$, Kang $\mathrm{HJ}$, Yoo CW et al. PLAG1, SOX10, and Myb Expression in Benign and Malignant Salivary Gland Neoplasms. J Pathol Transl Med 2019; 53: 23-30.
42. Tsuta K, Raso MG, Kalhor $\mathrm{N}$ et al. Sox10-positive sustentacular cells in neuroendocrine carcinoma of the lung. Histopathology 2011; 58: 276-285.

43. Nounou MI, Elamrawy F, Ahmed N et al. Breast cancer: conventional diagnosis and treatment modalities and recent patents and technologies. Breast Cancer: Basic and Clinical Research 2015; 9: 17-34.

44. Stockman DL, Miettinen M, Suster S et al. Malignant gastrointestinal neuroectodermal tumor: clinicopathologic, immunohistochemical, ultrastructural, and molecular analysis of 16 cases with a reappraisal of clear cell sarcoma-like tumors of the gastrointestinal tract. The American Journal of Surgical Pathology 2012; 36: 857-868.

45. Li J, Shen J, Wang K et al. The roles of SOX family genes in sarcoma. Current Drug Targets 2016; 17: 1761-1772.

46. Bakos RM, Maier T, Besch R et al. Nestin and SOX9 and SOX10 transcription factors are coexpressed in melanoma. Experimental Dermatology 2010; 19: e89-94.

47. Yang G, Minasyan A, Gordon J et al. http://www.cellmarque.com/ cms/downloads/marketing/Academia/ACADEMIA_SOX10.pdf.

48. Intragumtornchai T, Bunworasate U, Wudhikarn $\mathrm{K}$ et al. Non-Hodgkin lymphoma in South East Asia: an analysis of the histopathology, clinical features, and survival from Thailand. Hematological Oncology 2018; 36: 28-36.

49. Zuo H, Wood WM, Sherafat A et al. Age-dependent decline in fate switch from NG2 cells to astrocytes after olig2 deletion. The Journal of Neuroscience 2018; 38: 2359-2371.

50. An S, Jang J, Min K et al. Granular cell tumor of the gastrointestinal tract: histologic and immunohistochemical analysis of 98 cases. Human Pathology 2015; 46: 813-819.

51. Chamberlain BK, McClain CM, Gonzalez RS et al. Alveolar soft part sarcoma and granular cell tumor: an immunohistochemical comparison study. Human Pathology 2014; 45: 1039-1044.

52. Miettinen M, McCue PA, Sarlomo-Rikala M et al. SOX10 - a marker for not only schwannian and melanocytic neoplasms but also myoepithelial cell tumors of soft tissue: a systematic analysis of 5134 tumors. The American Journal of Surgical Pathology 2015; 39: 826-835. 